\title{
Cystatin C Enhances Glomerular Filtration Rate Estimating Equations in Kidney Transplant Recipients
}

\author{
Aleksandra Kukla ${ }^{a}$ Naim Issa ${ }^{a}$ Scott Jackson ${ }^{a}$ Richard Spong ${ }^{a}$ \\ Meredith C. Foster $^{b}$ Arthur J. Matas ${ }^{c}$ Michael S. Mauer ${ }^{d}$ \\ John H. Eckfeldt ${ }^{\mathrm{e}}$ Hassan N. Ibrahim ${ }^{\mathrm{a}}$ \\ a Division of Renal Diseases and Hypertension, Department of Medicine, University of Minnesota, \\ Minneapolis, Minn., b Department of Epidemiology, Johns Hopkins Bloomberg School of Public Health, \\ Baltimore, Md., and Departments of ' Surgery, 'Pediatrics, and 'Laboratory Medicine and Pathology, \\ University of Minnesota, Minneapolis, Minn., USA
}

\section{Key Words}

Glomerular filtration rate $\cdot$ Chronic kidney disease epidemiology collaboration - Modification of Diet in Renal Disease $\cdot$ Cystatin C $\cdot$ Kidney transplantation

\begin{abstract}
Background: The glomerular filtration rate (GFR) estimating equation incorporating both cystatin $C$ and creatinine perform better than those using creatinine or cystatin $C$ alone in patients with reduced GFR. Whether this equation performs well in kidney transplant recipients cross-sectionally, and more importantly, over time has not been addressed. Methods: We analyzed four GFR estimating equations in participants of the Angiotensin II Blockade for Chronic Allograft Nephropathy Trial (NCT 00067990): Chronic Kidney Disease Epidemiology Collaboration equations based on serum cystatin C and creatinine (eGFR (CKD-EPI-Creat+CysC)), cystatin C alone (eGFR (CKD-EPI-CysC)), creatinine alone (eGFR (CKDEPI-Creat)) and the Modification of Diet in Renal Disease study equation (eGFR (MDRD)). lothalamate GFR served as a
\end{abstract}

standard (mGFR). Results: mGFR, serum creatinine, and cystatin C shortly after transplant were $56.1 \pm 17.0 \mathrm{ml} /$ $\mathrm{min} / 1.73 \mathrm{~m}^{2}, 1.2 \pm 0.4 \mathrm{mg} / \mathrm{dl}$, and $1.2 \pm 0.3 \mathrm{mg} / \mathrm{l}$ respectively. eGFR (CKD-EPI-Creat+CysC) was most precise $\left(R^{2}=0.50\right)$ but slightly more biased than eGFR (MDRD); $9.0 \pm 12.7$ versus $6.4 \pm 15.8 \mathrm{ml} / \mathrm{min} / 1.73 \mathrm{~m}^{2}$, respectively. This improved precision was most evident in recipients with mGFR $>60 \mathrm{ml} /$ $\mathrm{min} / 1.73 \mathrm{~m}^{2}$. For relative accuracy, eGFR (MDRD) and eGFR (CKD-EPI-Creat+CysC) had the highest percentage of estimates falling within $30 \%$ of mGFR; 75.8 and $68.9 \%$, respectively. Longitudinally, equations incorporating cystatin $C$ most closely paralleled the change in mGFR. Conclusion: eGFR (CKD-EPI-Creat+CysC) is more precise and reflects GFR change over time reasonably well. eGFR (MDRD) had superior performance in recipients with MGFR between 30 and $60 \mathrm{ml} / \mathrm{min} / 1.73 \mathrm{~m}^{2}$.

(c) 2014 S. Karger AG, Basel

A.K. and N.I. contributed equally to this work and are considered first authors.

\section{KARGER}

E-Mail karger@karger.com www.karger.com/ajn
(C) 2014 S. Karger AG, Basel

0250-8095/14/0391-0059\$39.50/0
Hassan N. Ibrahim, MD, MS

Division of Renal Diseases and Hypertension Department of Medicine, University of Minnesota 717 Delaware Street, S.E., Minneapolis, MN 55414 (USA)

E-Mail ibrah007@umn.edu 


\section{Introduction}

The shortcomings of creatinine-based equations to estimate glomerular filtration rate (eGFR) in kidney transplant recipients are well recognized. Cystatin $C$ possesses several features of an almost ideal marker of kidney function as it is freely filtered and almost completely catabolized in the proximal tubule with no significant secretion or reabsorption [1]. The equation incorporating both creatinine and cystatin $\mathrm{C}$ has been shown to be more precise in people with chronic kidney disease (CKD) as well as in those with normal GFR [2-4]. The performance of cystatin C-based equations has been previously tested in kidney transplant recipients and yielded mixed results [5-9]. We studied the newly introduced creatinine, cystatin C and cystatin $\mathrm{C}+$ creatinine-based equations by comparing their cross-sectional and longitudinal performance against iothalamate GFR in kidney transplant recipients. We hypothesized that the combined use of these two markers in estimating GFR will improve upon equations utilizing either marker alone.

\section{Methods}

This analysis was performed using data and stored samples derived from the National Institutes of Health sponsored Angiotensin II Blockade for Chronic Allograft Nephropathy Trial (clinicaltrials. gov NCT 00067990). In this 5-year-long trial, 153 kidney transplant recipients were randomly allocated to placebo or losartan at $58 \pm 34$ days after receiving a living or deceased donor kidney transplant. Recipients were transplanted either at Hennepin County Medical Center (HCMC) or the University of Minnesota (UMN) - both in Minneapolis, Minn., USA. The details of the trial and its main results are described elsewhere [10]. Recipients transplanted at HCMC were on steroids containing immunosuppression and received Solu-Medrol ${ }^{\circledR} 500 \mathrm{mg}$ i.v. $\times 3$ doses at the time of transplant followed by prednisone tapered down to a maintenance dose of $12 \mathrm{mg} /$ day by 6 months. For maintenance therapy they received a calcineurin inhibitor, mycophenolate mofetil and prednisone. In contrast, those transplanted at UMN received only a 5-day course of steroids in tapered doses (methylprednisolone $500 \mathrm{mg}$ given in the operating room followed by prednisone: $1 \mathrm{mg} / \mathrm{kg}$ on postoperative day $1,0.5$ $\mathrm{mg} / \mathrm{kg}$ on days 2 and 3, and $0.25 \mathrm{mg} / \mathrm{kg}$ on days 4 and 5) at the time of transplant. Recipients on steroids prior to transplantation remained on their previous maintenance dose. Rejection episodes were treated with steroids and a rapid taper (methylprednisolone $500 \mathrm{mg} \times 3$ days followed by prednisone tapered over 10-14 days down to $5 \mathrm{mg}$ daily indefinitely) or Thymoglobulin ${ }^{\circledR}$ for 7-10 days for steroid-resistant or histologically moderate-severe rejection episodes and remained on maintenance steroids as well. For maintenance therapy, patients received a combination of a calcineurin inhibitor (cyclosporine or tacrolimus) with mycophenolate mofetil or sirolimus. All recipients were on single-strength trimethoprim-sulfamethoxazole and no patients were prescribed cimetidine.
All participants underwent baseline and annual measurement of GFR using iothalamate clearance (mGFR) at randomization. Subsequently, five more GFR measurements were performed with the second mGFR at 11-13 months after randomization and yearly thereafter up to 5 years posttransplantation. Iothalamate GFR was administered as an intravenous loading dose followed by maintenance infusion. All recipients received a water load of $10 \mathrm{ml} / \mathrm{kg}$ to maintain urine flow rates at $>3 \mathrm{ml} / \mathrm{kg} / \mathrm{h}$. If this flow rate was not achieved, an additional oral water load of $5 \mathrm{ml} / \mathrm{kg}$ was given. Five timed urine and plasma collections were performed and the average was calculated. The coefficient of variation of mGFR was $<8 \%$.

\section{Measurement of Serum Creatinine and Cystatin C Levels}

Serum creatinine and serum cystatin $C$ were obtained on the morning of the mGFR and after an 8-12 h fast. Serum creatinine was measured at the same laboratory and calibrated to IDMStraceable creatinine as described elsewhere [11]. Cystatin $C$ was measured using the PENIA cystatin C kit on a ProSpec nephelometer (Siemens) in batches in 2006, 2009 and 2011. To account for drifting of cystatin C levels related to different years of measurement previously observed and described in detail [12], we used data from 169 recipients who had two assays performed in both 2006 and 2011, and 125 recipients who had two assays performed in both 2009 and 2011 to perform the standardization. For the 169 recipients, ordinary least squares regression modeling resulted in cystatin $C($ new $)=0.1842+0.6806 \cdot$ cystatin $C($ old $)$. For the 125 recipients, it resulted in cystatin $C$ (new) $=0.0876+0.8705 \cdot$ cystatin C (old). In both cases the cystatin $\mathrm{C}$ values used in the regression were highly correlated $(r=0.95$ and 0.98$)$. These cystatin $C$ values were then multiplied by 1.12 to bring the measured values into alignment with the ERM DA-471/IFCC international cystatin $\mathrm{C}$ reference material which is highly recommended for use in the CKD-EPI equations published in 2012 [4].

\section{GFR Estimating Equations}

eGFR was calculated using equations that are designed for use of IDMS-traceable creatinine and ERM DA-471/IFCC standardized cystatin C measurement: Modification of Diet in Renal Disease (eGFR (MDRD)) [13], the Chronic Kidney Disease Epidemiology Collaboration IDMS-traceable creatinine-based equation (eGFR (CKD-EPI-Creat)), the CKD EPI cystatin C equation (eGFR (CKD-EPI-CysC)), and CKD EPI creatinine + cystatin C equation (eGFR (CKD-EPI-Creat+CysC)) as outlined in the supplementary table (www.karger.com/doi/10.1159/357594) [4].

\section{Statistical Analysis}

We assessed the performance of these four eGFR equations against mGFR by studying bias - the average prediction error $=$ $\Sigma($ eGFR - mGFR)/n; relative bias - percent deviation from mGFR; correlation - the value of $\mathrm{R}^{2}$ and RMSE from the Deming linear regression of eGFR on $\mathrm{mGFR}$ and interquartile range of differences, and relative accuracy - the percent of eGFR values falling within 10 and $30 \%$ of mGFR in all recipients, recipients with $\mathrm{mGFR} 30-60 \mathrm{ml} / \mathrm{min} / 1.73 \mathrm{~m}^{2}$ and recipients with $\mathrm{mGFR} \geq 60 \mathrm{ml} /$ $\mathrm{min} / 1.73 \mathrm{~m}^{2}$. Recipients with $\mathrm{mGFR}<30 \mathrm{ml} / \mathrm{min} / 1.73 \mathrm{~m}^{2}$ were not included due to infrequent occurrence of this event. For each equation a Bland-Altman plot, Deming regression curve and residual density and histogram plot were constructed. These plots together show the variation of bias according to the level of mea-
60

Am J Nephrol 2014;39:59-65

DOI: $10.1159 / 000357594$
Kukla/Issa/Jackson/Spong/Foster/Matas/ Mauer/Eckfeldt/Ibrahim 
sured GFR. Deming regression is favored over ordinary least squares regression since there is error in both measured and estimated GFR.

Mean values (along with 95\% confidence intervals (CI)) were plotted for mGFR and each eGFR equation over time. A linear mixed model was used to predict bias over time. In this model, a random intercept was utilized with a common slope and an autoregressive covariance structure.

To evaluate whether cystatin $C$ and creatinine levels can be influenced by other factors, we fit a linear regression model at baseline to measure the effect of multiple covariates using backwards selection. The following covariates were considered: treatment (losartan or placebo), donor and recipient demographics, HLA matches, urine electrolytes, mGFR, use of prednisone, cyclosporine, tacrolimus, mycophenolate mofetil, trimethoprim-sulfamethoxazole, sirolimus and diuretics. GFR estimating equations were compared in recipients who were on maintenance steroids to those who were not using a two-sample $t$ test. All statistical analyses were performed using SAS version 9.3 (Cary, N.C., USA).

\section{Results}

153 recipients underwent iothalamate GFR measurement $58 \pm 34$ days after transplantation. Mean age at first GFR measurement was $48.9 \pm 12.4$ years. The majority of recipients were male $(61.4 \%)$ and white $(93.5 \%)$. Mean BMI was 26.4 , and $39.2 \%$ of patients were diabetic. Systolic and diastolic blood pressures at baseline were $130.5 \pm$ 18.3 and $73.5 \pm 10.7 \mathrm{~mm} \mathrm{Hg}$ respectively (table 1). Only 42 recipients $(27.5 \%)$ were on steroids. Most received living donor transplant (70.6\%) and most of the donors were females (54.2\%). Mean donor age was $40.3 \pm 12.1$ years and mean iothalamate GFR for recipients was $56.1 \pm$ $17.0 \mathrm{ml} / \mathrm{min} / 1.73 \mathrm{~m}^{2}$.

\section{Cross-Sectional Performance of GFR Estimating Equations}

The overall bias, relative bias, precision and accuracy of the four equations for all patients as well as based on the level of mGFR $30-60$ and $\geq 60 \mathrm{ml} / \mathrm{min} / 1.73 \mathrm{~m}^{2}$ at the time of randomization are shown in table 2. eGFR CKDEPI (Creat+CysC) equation had a bias of $9.0 \pm 12.7 \mathrm{ml} /$ $\mathrm{min} / 1.73 \mathrm{~m}^{2}$, which was comparable to that of eGFR (CKD-EPI-CysC), but larger than eGFR (MDRD). Relative bias was the lowest for combined equation and eGFR MDRD $(26.5 \pm 27.1$ and $25.5 \pm 29.8 \%$, respectively). eGFR (CKD-EPI-Creat+CysC) equation was by far most precise $\left(\mathrm{R}^{2}=0.50\right)$. The three other equations had smaller and similar values with a range of $0.37-0.38$. When stratified by mGFR, all equations studied performed better in terms of bias, precision and accuracy in recipients with $\mathrm{mGFR} \geq 60$ versus $30-60 \mathrm{ml} / \mathrm{min} / 1.73 \mathrm{~m}^{2}$.
Table 1. Demographics at baseline

\begin{tabular}{lc}
\hline Variable & Mean (SD) or \% \\
\hline Recipients & \\
$\quad$ Age at GFR, years & $48.9(12.4)$ \\
$\quad$ Male & 61.4 \\
$\quad$ Black & 6.5 \\
$\quad$ BMI, kg/m ${ }^{2}$ & $26.4(5.1)$ \\
$\quad$ Diabetic & 39.2 \\
Donors & \\
$\quad$ Living & 70.6 \\
$\quad$ Age at GFR, years & $40.3(12.1)$ \\
$\quad$ Male & 45.8 \\
$\quad$ Black & 5.2 \\
Systolic blood pressure, mm Hg & $130.5(18.3)$ \\
Diastolic blood pressure, $\mathrm{mm} \mathrm{Hg}$ & $73.5(10.7)$ \\
Serum creatinine, mg/dl & $1.2(0.4)$ \\
Serum cystatin C, mg/l & $1.2(0.3)$ \\
Albumin-creatinine ratio, mg/g & $77.9(167.0)$ \\
mGFR, ml/min/1.73 m ${ }^{2}$ & $56.1(17.0)$ \\
Steroid maintenance & 27.5 \\
\hline
\end{tabular}

In those with $\mathrm{mGFR} 30-60 \mathrm{ml} / \mathrm{min} / 1.73 \mathrm{~m}^{2}$, eGFR (MDRD) had the lowest bias and the highest accuracy, with $70.9 \%$ estimates falling within $30 \%$ of mGFR. In recipients with $\mathrm{mGFR} \geq 60 \mathrm{ml} / \mathrm{min} / 1.73 \mathrm{~m}^{2}$, eGFR (CKD-EPI-Creat+CysC) equation had a relatively low bias of $2.9 \mathrm{ml} / \mathrm{min} / 1.73 \mathrm{~m}^{2}$ and precision $\left(\mathrm{R}^{2}\right)$ of 0.39 , which was comparable to eGFR (MDRD) and better than other equations. Notably, it was the most accurate with $93 \%$ of estimates falling within $30 \%$ of mGFR.

Figure 1 depicts the Bland-Altman and Deming regression plots, as well as a density curve and histogram of the residuals (biases) for each equation. These figures graphically demonstrate that the eGFR (MDRD) and eGFR (CKD-EPI-Creat) equations underestimated mGFR at lower mGFR levels and overestimated it at higher mGFR levels. Bland-Altman plots also show that eGFR (CKD-EPI-Creat+CysC) had the lowest variability with respect to its bias (fig. 1a). The combined histogram/density plot shows how well the residuals (i.e. biases) follow a normal distribution. We formally examined the distribution of the biases using the Shapiro-Wilk test and found that they were normal for both cystatin-C based equations ( $\mathrm{p}=0.64$ and $\mathrm{p}=0.26$ for eGFR (CKDEPI-CysC) and eGFR (CKD-EPI-Creat+CysC), respectively). Overall, this shows that the biases of the cystatin C-based equations are not dependent on their estimated values. 
Table 2. Overall performance of GFR estimating equations

\begin{tabular}{|c|c|c|c|c|c|c|c|c|}
\hline $\begin{array}{l}\text { Recipients } \\
\text { eGFR method }\end{array}$ & $\begin{array}{l}\text { Mean (SD), } \\
\mathrm{ml} / \mathrm{min} / 1.73 \mathrm{~m}^{2}\end{array}$ & Bias (SD) & IQR & $\begin{array}{l}\text { Relative bias, } \\
\%\end{array}$ & $\mathrm{R}^{2 * *}$ & RMSE & $\begin{array}{l}\text { Within } 10 \% \\
\text { of mGFR }\end{array}$ & $\begin{array}{l}\text { Within } 30 \% \\
\text { of mGFR }\end{array}$ \\
\hline \multicolumn{9}{|l|}{$\operatorname{All}(n=153)$} \\
\hline eGFR (CKD-EPI-CysC) & $64.7(16.7)$ & $8.6(15.0)$ & 18.6 & $29.0(30.8)$ & 0.37 & 11.8 & $29.1 \%$ & $66.9 \%$ \\
\hline eGFR (CKD-EPI-Creat) & $67.9(20.0)$ & $11.8(16.6)^{*}$ & 18.1 & $31.5(34.5)$ & 0.37 & 12.3 & $28.1 \%$ & $65.4 \%$ \\
\hline eGFR (MDRD) & $62.5(18.7)$ & $6.4(15.8)^{*}$ & 16.4 & $25.5(29.8)$ & 0.38 & 12.0 & $32.7 \%$ & $75.8 \%$ \\
\hline \multicolumn{9}{|l|}{$30 \leq m G F R<60(n=86)$} \\
\hline eGFR (CKD-EPI-Creat+CysC) & $59.4(12.5)$ & $12.6(11.2)$ & 13.5 & $31.2(24.3)$ & 0.22 & 7.0 & $15.3 \%$ & $55.3 \%$ \\
\hline eGFR (CKD-EPI-CysC) & $59.1(14.3)$ & $12.3(13.8)$ & 16.0 & $34.1(25.7)$ & 0.12 & 7.6 & $18.8 \%$ & $56.5 \%$ \\
\hline eGFR (CKD-EPI-Creat) & $62.5(16.6)$ & $15.8(15.5)$ & 20.6 & $36.7(37.1)$ & 0.14 & 7.5 & $27.9 \%$ & $55.8 \%$ \\
\hline eGFR (MDRD) & $57.5(15.3)$ & $10.8(14.4)$ & 17.3 & $28.5(32.8)$ & 0.13 & 7.5 & $33.7 \%$ & $70.9 \%$ \\
\hline \multicolumn{9}{|l|}{$m G F R>60(n=61)$} \\
\hline
\end{tabular}

* Significant pairwise comparison. ${ }^{* *} \mathrm{R}^{2}$ and RMSE from Deming regression.

\section{Longitudinal Performance of eGFR Equations}

The number of recipients who underwent measured GFR and had creatinine and cystatin $\mathrm{C}$ measured decreased from 153 (visit 1) to 85 (visit 6). This decrease in number is due to the fact that some recipients reached ESRD or have left the study. Figure 2 shows mean estimated and mGFR along with 95\% CI at baseline and yearly posttransplant. Measured GFR at 5 years was $53.7 \pm$ 16.8; eGFR (CKD-EPI-Creat+CysC) most closely followed the trend of mGFR. Using a mixed model, we also considered how the bias for each equation changed over time. In the case of both eGFR (CKD-EPI-CysC) and eGFR (CKD-EPI-Creat+CysC) this change was not significant ( $\mathrm{p}=0.60$ and $\mathrm{p}=0.06$ ), suggesting that the bias did not depend on the year of measurement (fig. 2).

\section{Effect of Multiple Covariates on Creatinine and Cystatin C Levels}

In multivariate analysis, we found that an increase in mGFR resulted in a decrease of cystatin $\mathrm{C}$ (estimate $=$ $-0.009, \mathrm{p}<0.001$ ), and receiving maintenance steroids resulted in an increase of cystatin $\mathrm{C}$ levels (estimate $=$ $0.119, \mathrm{p}=0.003$ ). Cystatin C-based eGFR values were not significantly different in recipients who used steroids eGFR (CKD-EPI-CysC) was $61 \pm 15.8$ vs. $66.1 \pm 16.9 \mathrm{ml} /$ $\mathrm{min} / 1.73 \mathrm{~m}^{2}$ for recipients on and off steroids, respectively, $\mathrm{p}=0.10$, and eGFR (CKD-EPI-Creat+CysC was
$63.9 \pm 17.1$ vs. $65.6 \pm 16.0 \mathrm{ml} / \mathrm{min} / 1.73 \mathrm{~m}^{2}$ for recipients on and off steroids, respectively, $\mathrm{p}=0.57$ ). A lower mGFR, CNI inhibitors use, male gender, lower age at GFR, higher donor age and higher BMI were all associated with a significant increase in creatinine ( $p<0.05$ in all cases). Steroids maintenance did not influence levels of creatinine or measured GFR.

\section{Discussion}

Our results show that eGFR (CKD-EPI-Creat+CysC) has some advantages over other equations, as it has higher precision, comparable bias to other equations, and more accurately reflects the changes of mGFR over time. Importantly, its bias is not dependent on eGFR values. It does not, however, perform better then eGFR MDRD in recipients with mGFR $30-60 \mathrm{ml} / \mathrm{min} / 1.73 \mathrm{~m}^{2}$. Performance of combined creatinine and cystatin $C$ formula was previously reported by Masson et al. [14]. While the latter analysis showed smaller bias and better precision of eGFR (CKD-EPI-Creat+CysC) than what we observed in our analysis, that could be related to the differences in mean measured GFR: $50.1 \pm 18.6 \mathrm{ml} / \mathrm{min} / 1.73 \mathrm{~m}^{2}$ in the Masson et al. study versus $56.1 \pm 17.0 \mathrm{ml} / \mathrm{min} / 1.73 \mathrm{~m}^{2}$ in our study $(\mathrm{p}<0.01)$, differences in measured GFR methodologies ( 2 time points inulin clearance versus 5 iothal-
62

Am J Nephrol 2014;39:59-65 DOI: $10.1159 / 000357594$
Kukla/Issa/Jackson/Spong/Foster/Matas/ Mauer/Eckfeldt/Ibrahim 


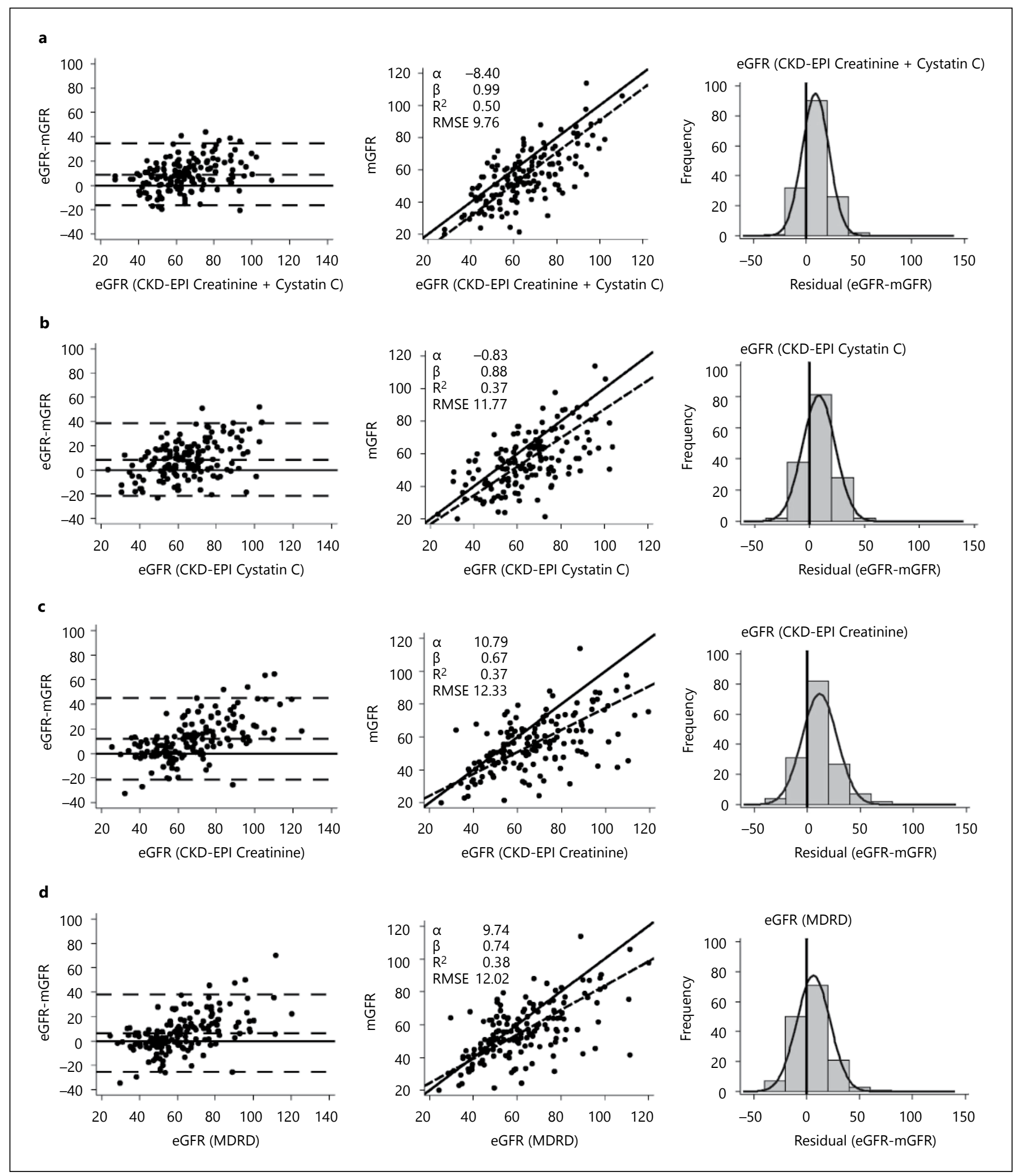

Fig. 1. Bland-Altman plots showing the distribution of errors in estimation of mGFR with eGFR, Deming regression plots with precision ( $\mathrm{R}^{2}$ and $\mathrm{RMSE}$ ), histograms and density curves of the distribution of the difference (bias) between eGFR and mGFR for (a) CKD-EPI Creatinine + Cystatin C, (b) CKD-EPI Cystatin C, (c) CKD-EPI Creatinine, and (d) MDRD.
GFR Estimation in Kidney Transplant Recipients
Am J Nephrol 2014;39:59-65 DOI: $10.1159 / 000357594$ 


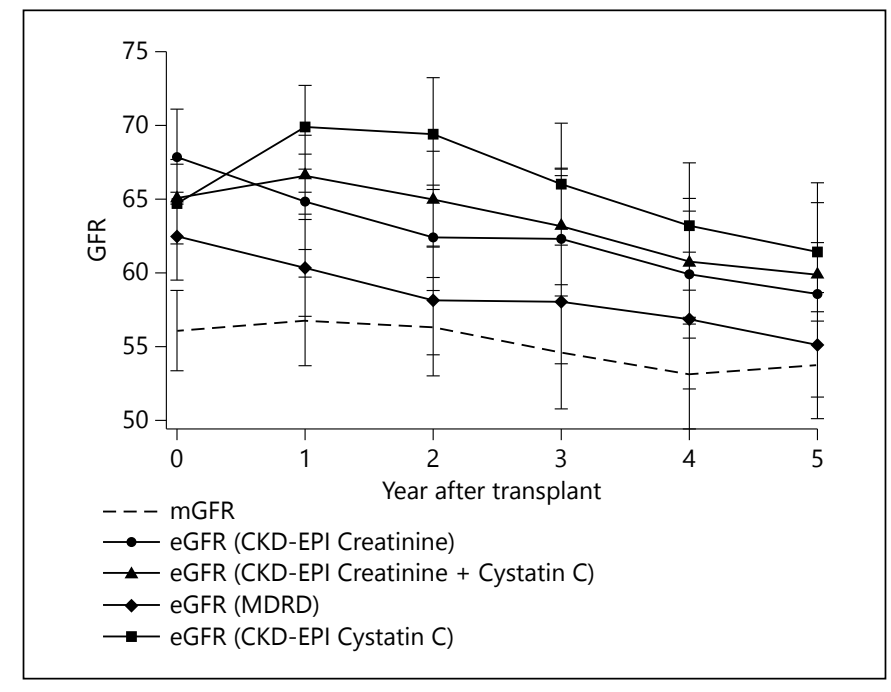

Fig. 2. Mean measured and estimated GFR (along with 95\% CI) at baseline and yearly posttransplant (the distance between the plot for each eGFR and mGFR represents the bias at the given year).

amate clearance), and most importantly, the fact that we standardized cystatin $\mathrm{C}$ levels using the conversion equation developed based on our own data to account for drifting over time. Other previously published data reported mixed results in terms of performance of cystatin C equations in kidney transplant recipients. Poge et al. [7] showed superior diagnostic performance of three cystatin C-based equations (Larsson, Hoek, Filler) compared to the MDRD study equation. Median measured GFR was $37 \mathrm{ml} / \mathrm{min} / 1.73 \mathrm{~m}^{2}$, which was $15 \mathrm{ml} / \mathrm{min} / 1.73 \mathrm{~m}^{2}$ lower than in our analysis. Another study showed an improved ability of cystatin C-based equations to classify recipients into correct CKD stage [8]. Lastly, Zahran et al. [9] did not confirm the superiority of cystatin $C$ equations in recipients with measured GFR of $46 \pm 20.9 \mathrm{ml} / \mathrm{min} / 1.73 \mathrm{~m}^{2}$. This inconsistency may be related to the lack of calibration of creatinine and cystatin $C$ values in previous studies and obviously the heterogenicity of patients and the wide range of GFR. More studies including equations with standardized levels of cystatin $\mathrm{C}$ are needed to increase our understanding of applicability of cystatin C incorporating equations in different populations.

The longitudinal performance of GFR estimates in recipients with preserved kidney function provides clinically important information. Similar but much smaller studies have been previously done in non-transplant populations. Evaluation of estimates to correctly detect kidney function decline at higher levels of GFR was performed in 36 patients with Fabry's disease [3]. The authors tested eleven cystatin $C$, creatinine and $\beta$ trace protein equations against iothalamate GFR. Measured GFR declined only in men from median 97.3 to $79.3 \mathrm{ml} / \mathrm{min} / 1.73 \mathrm{~m}^{2}$. In this study, equations based on cystatin $\mathrm{C}$ alone did not accurately predict measured GFR decline, mainly due to the significant underestimation of measured GFR at the higher levels $\left(>125 \mathrm{ml} / \mathrm{min} / 1.73 \mathrm{~m}^{2}\right)$. The equation incorporating creatinine and cystatin $\mathrm{C}$ had the best performance. In our study, eGFR (CKD-EPI-CysC) and eGFR (CKD$\mathrm{EPI}-\mathrm{Creat}+\mathrm{CysC}$ ) were the only equations with a bias that did not significantly change over time.

A maintenance steroid was associated with higher levels of cystatin C, consistent with other observations [15, 16]. Potential explanation may lay in the promoter-mediated steroid-induced increase in transcription of the cystatin $\mathrm{C}$ gene, as previously shown in vitro [17]. Nevertheless, there was no significant difference in eGFR for recipients on and off steroids. However, we suggest that equations incorporating cystatin $\mathrm{C}$ levels be used with caution in recipients undergoing treatment with high doses of steroids. We found multiple factors which influenced creatinine levels, and this could potentially explain decreased performance of creatinine-only based equations.

While this analysis has the distinct advantages of utilizing standardized creatinine and cystatin $\mathrm{C}$ assays and provides insight into the ability of these equations to detect GFR change over time, it suffers from the fact that the majority of participants were white. Obviously, a large cohort is needed to address this area more credibly. Additionally, cystatin $\mathrm{C}$ measurements were not done at the same time, which can impact reproducibility of results due to the drifting of cystatin C levels. We attempted to correct for that by regression adjustments and standardized cystatin $\mathrm{C}$ levels in alignment with international cystatin $\mathrm{C}$ reference material.

We conclude that the combined serum creatinine and serum cystatin $\mathrm{C}$ estimate is more precise than either marker alone (especially in recipients with better preserved kidney function) for estimating GFR and reflects GFR change over time reasonably well. eGFR (MDRD), however, had superior performance to other equations in those with $\mathrm{mGFR}$ between 30 and $60 \mathrm{ml} / \mathrm{min} / 1.73 \mathrm{~m}^{2}$ and therefore we recommend this formula for the routine use in clinical practice.

\section{Disclosure Statement}

The authors have no conflicts of interest to disclose. Dr. Foster was supported in part by NIH/National Heart, Lung and Blood Institute Cardiovascular Epidemiology training grant T32HL007024.
Kukla/Issa/Jackson/Spong/Foster/Matas/ Mauer/Eckfeldt/Ibrahim 


\section{References}

$>1$ Filler G, Bokenkamp A, Hofmann W, Le Bricon T, Martinez-Bru C, Grubb A: Cystatin C as a marker of GFR - history, indications, and future research. Clin Biochem 2005;38:1-8.

-2 Stevens LA, Coresh J, Schmid CH, Feldman HI, Froissart M, Kusek J, Rossert J, Van Lente F, Bruce RD 3rd, Zhang YL, Greene T, Levey AS: Estimating GFR using serum cystatin C alone and in combination with serum creatinine: a pooled analysis of 3,418 individuals with CKD. Am J Kidney Dis 2008;51:395406.

>3 Rombach SM, Baas MC, ten Berge IJ, Krediet RT, Bemelman FJ, Hollak CE: The value of estimated GFR in comparison to measured GFR for the assessment of renal function in adult patients with Fabry disease. Nephrol Dial Transplant 2010;25:2549-2556.

-4 Inker LA, Schmid CH, Tighiouart H, Eckfeldt JH, Feldman HI, Greene T, Kusek JW, Manzi J, Van Lente F, Zhang YL, Coresh J, Levey AS, CKD-EPI Investigators: Estimating glomerular filtration rate from serum creatinine and cystatin C. N Engl J Med 2012;367:20-29.

5 Risch L, Huber AR: Assessing glomerular filtration rate in renal transplant recipients by estimates derived from serum measurements of creatinine and cystatin C. Clin Chim Acta 2005;356:204-211.
-6 Maillard N, Mariat C, Bonneau C, Mehdi M, Thibaudin L, Laporte S, Alamartine E, Chamson A, Berthoux F: Cystatin C-based equations in renal transplantation: moving toward a better glomerular filtration rate prediction? Transplantation 2008;85:1855-1858.

7 Poge U, Gerhardt T, Stoffel-Wagner B, Palmedo H, Klehr HU, Sauerbruch T, Woitas RP: Cystatin C-based calculation of glomerular filtration rate in kidney transplant recipients. Kidney Int 2006;70:204-210.

$>8$ White C, Akbari A, Hussain N, Dinh L, Filler G, Lepage N, Knoll GA: Chronic kidney disease stage in renal transplantation classification using cystatin $\mathrm{C}$ and creatinine-based equations. Nephrol Dial Transplant 2007;22: 3013-3020.

-9 Zahran A, Qureshi M, Shoker A: Comparison between creatinine and cystatin C-based GFR equations in renal transplantation. Nephrol Dial Transplant 2007;22:2659-2668.

$>10$ Ibrahim HN, Jackson S, Connaire J, Matas A, Ney A, Najafian B, West A, Lentsch N, Ericksen J, Bodner J, Kasiske B, Mauer M: Angiotensin II blockade in kidney transplant recipients. J Am Soc Nephrol 2013;24:320-327.

11 Kukla A, El-Shahawi Y, Leister E, Kasiske B, Mauer M, Matas A, Ibrahim HN: GFR-estimating equations in kidney transplant recipients on a steroid-free regimen. Nephrol Dial Transplant 2010;25:1653-1661.

-12 Inker LA, Eckfeldt J, Levey AS, LeiendeckerFoster C, Rynders G, Manzi J, Waheed S, Coresh J: Expressing the CKD-EPI (Chronic Kidney Disease Epidemiology Collaboration) cystatin $\mathrm{C}$ equations for estimating GFR with standardized serum cystatin $\mathrm{C}$ values. Am J Kidney Dis 2011;58:682-684.
3 Levey AS, Coresh J, Greene T, Stevens LA, Zhang YL, Hendriksen S, Kusek JW, Van Lente F, Chronic Kidney Disease Epidemiology Collaboration: Using standardized serum creatinine values in the Modification of Diet in Renal Disease study equation for estimating glomerular filtration rate. Ann Intern Med 2006;145:247-254.

14 Masson I, Maillard N, Tack I, Thibaudin L, Dubourg L, Delanaye P, Cavalier E, Bonneau C, Kamar N, Morelon E, Moranne O, Alamartine E, Mariat C: GFR estimation using standardized cystatin $C$ in kidney transplant recipients. Am J Kidney Dis 2013;61:279-284.

15 Risch L, Herklotz R, Blumberg A, Huber AR: Effects of glucocorticoid immunosuppression on serum cystatin $\mathrm{C}$ concentrations in renal transplant patients. Clin Chem 2001;47: 2055-2059.

-16 Poge U, Gerhardt T, Bokenkamp A, StoffelWagner B, Klehr HU, Sauerbruch T, Woitas RP: Time course of low molecular weight proteins in the early kidney transplantation period - influence of corticosteroids. Nephrol Dial Transplant 2004;19:2858-2863.

17 Bjarnadottir M, Grubb A, Olafsson I: Promoter-mediated, dexamethasone-induced increase in cystatin C production by HeLa cells. Scand J Clin Lab Invest 1995;55:617-623. 